\title{
EXTENSIONS OF HAAR MEASURE TO RELATIVELY LARGE NONMEASURABLE SUBGROUPS(')
}

BY

\author{
H. LEROY PETERSON
}

\begin{abstract}
Let $G$ be a locally compact group, with $\lambda$ a left Haar measure on $G$. A subgroup is large if it has finite index; a relatively large subgroup of $G$ is a large subgroup of an open subgroup. In $\$ 1$ we have an existence theorem for relatively large nonopen subgroups, and we observe that such subgroups are not $\lambda$-measurable. This motivates the development, in \$2, of a left-invariant countably additive extension $\lambda^{+}$of $\lambda$, which includes in its domain all unions of left translates of a given relatively large subgroup $K$. For an arbitrarily chosen family $\Re_{I}$ of relatively large subgroups of $G$, we define (in 83) a finitely additive measure $\lambda_{I}^{+}$such that, for any $K \in \mathscr{K}_{I}, \lambda_{I}^{+}$ is an extension of the corresponding $\lambda^{+}$defined in $\$ 2$. An example shows that $\lambda_{I}^{+}$need not be countably additive. Finally, in $\$ 4$, we observe some aspects of the relationship between $\lambda^{+}$-measurable and $\lambda$-measurable functions, in the context of existing literature on extensions of Haar measure. In particular, we generalize the well-known proposition that $\lambda$-measurable characters are continuous.
\end{abstract}

\section{Introduction.}

1.1. Preliminaries. The symbol $G$ henceforth denotes a locally compact group; $\lambda$ denotes a (left) Haar measure on $G$ with domain $\Re$. More generally, if $H$ is any locally compact group, $\Re_{H}$ denotes the family of Haar-measurable subsets of $H$.

As basic references for group theory, topology, topological groups, and measure theory, we will use [13], [10], [2], and [4], respectively. (Readers using [1] as a reference will note that the definitions therein are not entirely consistent with those in [2] and [4]. In particular, $\mathfrak{N}$ may have locally $\lambda$-null members which are not $\lambda$-null. All locally compact groups referred to are assumed to be $T_{0}$, as in $\$ \S 9-26$ of [2].)

Received by the editors December 3, 1975.

AMS (MOS) subject classifications (1970). Primary 22D05, 28A70; Secondary 22B05, 28A25, $43 \mathrm{~A} 40$.

Key words and phrases. Subgroups of finite index, locally compact group, extension of Haar measure, sets of full outer measure, characters on an Abelian group.

(1) This research was partially supported by Indiana University Faculty Summer Fellowships awarded in 1972 and 1975.

- American Mathematical Society 1977 
By a left-invariant extension of $\lambda$ we mean an extension $\left(G, \mathfrak{T}^{+}, \lambda^{+}\right)$of the measure space $(G, \Re, \lambda)$, where $\lambda^{+}$is countably additive, nonnegative, and invariant under left translation. An invariant extension of $\lambda$ is a left-invariant extension in which $\mathfrak{T}^{+}$is closed under inversion and right (as well as left) translation of its members.

For any set $X, \mathcal{P}(X)$ denotes the power set of $X$. For any function $f$ whose domain contains $X, f \mid X$ denotes the restriction of $f$ to $X$. By a translate of a subset $S$ of $G$, we mean a set of the form $x S y$ for some $x, y \in G$. (Left and right translates are defined as usual.)

It is useful to observe that a subgroup $K$ of $G$ is relatively large if and only if $K$ has open closure in $G$ and $[\operatorname{cl}(K): K]$ is finite.

1.2. ThEOREM. Let $C$ be the component of e (the identity element) in $G$. Then:

(1) If $C$ is open, and compact or Abelian (or both), then every relatively large subgroup of $G$ is open.

(2) If $C$ is not open, and $G / C$ is a compact Abelian torsion group or a compact nonmetrizable group, then $G$ has at least $2^{c}$ large nonopen subgroups.

(3) If $G / C$ is not metrizable, or if $G / C$ has an infinite open, Abelian, torsion subgroup, then $G$ has at least $2^{c}$ relatively large nonopen subgroups.

Proof. (1) Suppose $K$ is a relatively large subgroup of $G$. Then $K$ contains every divisible subgroup of $\operatorname{cl}(K)$ and thus (as in (1.2) of [11]) $C \subset K$; if $C$ is open, this implies that $K$ is open also.

(2) See (2.5) and (2.6) of [11].

(3) In either case, $G$ has an open subgroup $H$ such that $C \subset H$ and $C / H$ is compact. We may apply (2) to this subgroup.

We shall now develop some properties of relatively large subgroups, to be used in the sequel. In 1.3-1.5, the local compactness of $G$ is not used.

1.3. THEOREM. Let $J$ be a subgroup of $G$ and $K$ a relatively large subgroup of $G$. Then $J$ is relatively large in $G$ if any of the following is true:

(1) $K \subset J$;

(2) $J$ is a relatively large subgroup of $K$;

(3) $J \cap K$ is a relatively large subgroup of $K$.

Proof. (1) Since $K$ has open closure in $G$, so does $J$. Let $H_{1}=\operatorname{cl}(K)$; then $\operatorname{cl}(J)=J H_{1}$, and $[\operatorname{cl}(J): J]$ is finite since $\left[J H_{1}: J\right]=\left[H_{1}: J \cap H_{1}\right] \leqslant\left[H_{1}\right.$ : $K]$.

(2) Since $J$ has open closure in $K$, there is a neighborhood $U$ of $e$ in $G$ such that $U \cap K \subset \operatorname{cl}(J)$. It follows that $U \cap \operatorname{cl}(K) \subset \operatorname{cl}(J)$; hence $\operatorname{cl}(J)$ is open in $G$. If $H_{2}=\mathrm{cl}(J)$, then $\left[H_{2}: J\right]$ is finite, for $\operatorname{cl}(K)=K H_{2}$ and

$$
\left[H_{2}: J\right]=\left[H_{2}: H \cap K\right]\left[H_{2} \cap K: J\right]=[\operatorname{cl}(K): K]\left[\operatorname{cl}_{k}(J): J\right] .
$$


(3) By case (2), $J \cap K$ is relatively large in $G$, and thus by case (1), $J$ is relatively large in $G$.

1.4. LEMMA. (1) The intersection of finitely many relatively large subgroups of $G$ is relatively large.

(2) Every conjugate of a relatively large subgroup of $G$ is relatively large.

(3) A relatively large subgroup of $G$, having only finitely many conjugates, contains a relatively large normal subgroup of $G$.

Proof. (1) It suffices to show that the intersection of two relatively large subgroups is relatively large. Let $K_{j}$ be a large subgroup of $U_{j}(j=1,2)$; then $K_{1} \cap K_{2}$ is a large subgroup of $U_{1} \cap U_{2}$, for

$$
\begin{aligned}
{\left[U_{1} \cap U_{2}: K_{1} \cap K_{2}\right] } & =\left[U_{1} \cap U_{2}: U_{1} \cap K_{2}\right]\left[U_{1} \cap K_{2}: K_{1} \cap K_{2}\right] \\
& \leqslant\left[U_{1}: K_{2}\right]\left[U_{1}: K_{1}\right] .
\end{aligned}
$$

(2) Observe that, for all $x \in G,\left[\operatorname{cl}\left(x K x^{-1}\right): x K x^{-1}\right]=[\operatorname{cl}(K): K]$.

(3) Follows from (1) and (2).

1.5. THEOREM. Let $J$ be a subgroup of $G$. Then the following statements are equivalent:

(1) $J$ contains a relatively large normal subgroup of $G$.

(2) $J$ contains a relatively large normal subgroup of a large subgroup of $G$.

(3) $J$ contains a relatively large subgroup of $G$ which has only finitely many conjugates in $G$.

Proof. Trivially, (1) implies (2). If $K$ is any normal subgroup of any large subgroup of $G, N_{G}(K)$ (the normalizer of $K$ ) is finite. Thus (2) implies (3). Finally, 1.4(3) states that (3) implies (1).

1.6. Comment. Motivation for Theorem 1.5 may be found below, in the statement of Theorem 2.3.

According to $1.3(2)$, the subgroups $J$ described in 1.5 are relatively large in $G$. If $G$ is compact, or more generally if every open subgroup of $G$ has finite index, then every relatively large subgroup of $G$ is large and has only finitely many conjugates.

1.7. Definition. A subset $S$ of $G$ has full outer measure if $\lambda F=0$ for every compact set $F$ such that $F \cap S=\varnothing$. More generally, let us say that $S$ has full outer measure in its closure if $\operatorname{cl}(S)$ is not locally null and $\lambda F=0$ for each compact set $F$ such that $F \subset \operatorname{cl}(S)$ and $F \cap S=\varnothing$.

1.8. LEMMA. Every translate of a relatively large nonopen subgroup $K$ of $G$ has full outer measure in its closure and is not Haar measurable.

Proof. Since $\mathrm{cl}(K)$ is an open set covered by finitely many translates of $K$, neither $K$ nor $\mathrm{cl}(K)$ is locally null. Lemma 1 of [13] implies that $K$ (and, thus, 
every translate of $K$ ) has full outer measure in its closure. $K$ contains no compact set of positive measure since each coset of $K$ in $\operatorname{cl}(K)$ has full outer measure in $\mathrm{cl}(K)$; since $K$ is not locally null, this implies that $K$ is not $\lambda$ measurable.

1.9. Corollary. Let $K$ be a relatively large subgroup of $G$. Suppose $M_{1}$ and $M_{2}$ are $\sigma$-finite members of $\Re \cap \mathcal{P}(\operatorname{cl}(K))$ and $M_{1} \cap K=M_{2} \cap K$. Then $\lambda M_{1}=\lambda M_{2}$.

Proof. Let $M=\left(M_{1} \backslash M_{2}\right) \cup\left(M_{2} \backslash M_{1}\right)$. If $F$ is a compact subset of $M, \lambda F$ $=0$ since $F \cap K=\varnothing$. Thus $\lambda M=0$, and $\lambda M_{1}=\lambda M_{2}$.

1.10. Definition. We say a subset of $G$ is $\sigma$-finite if it is contained in a $\sigma$ finite member of $\mathfrak{T}$. For any family $\mathbb{Q}$ of subsets of $G, \mathbb{Q}_{0}$ denotes the subfamily of $\sigma$-finite members of $Q$.

1.11. THEOREM. Let $S \subset G$. Then the following statements are equivalent:

(1) $S$ is $\sigma$-finite.

(2) If $U$ is any open subgroup of $G, S$ intersects only countably many (left or right) cosets of $U$.

(3) If $K$ is any relatively large subgroup of $G, S$ intersects only countably many cosets of $K$.

(4) There exists a $\sigma$-compact open subgroup $U$ of $G$ such that $S$ intersects only countably many translates of $G$.

(5) $\mathrm{cl}(S)$ is $\sigma$-compact.

Proof. It is a simple exercise to show that (1) implies (2), (2) is equivalent to (3), (2) implies (4), (4) implies (5), and (5) implies (1).

1.12. Corollary. A subset $S$ of $G$ is $\sigma$-finite if and only if every $\lambda$-measurable subset of $S$ is $\sigma$-finite.

Proof. It is trivial that any subset of a $\sigma$-finite set is $\sigma$-finite. Conversely, if $S$ is not $\sigma$-finite, the negation of 1.11(4), together with the proof of (16.14) of [2], shows that $S$ contains a closed subset $F$ of $G$, such that $F$ is not $\sigma$-finite. (In fact, $\lambda F=\infty$ but every $\sigma$-finite subset of $F$ has measure zero.)

\section{An extension of Haar measure.}

2.1. Notation. As before, $K$ is a (nonopen) relatively large subgroup of $G$. Let $A=G / K$, let $\left\{h_{j}: j=1,2, \ldots, n\right\}$ be a complete set of representatives for $\operatorname{cl}(K) / K$, and define $\mathfrak{T R}^{+}$to be the family of all subsets $S$ of $G$ such that, for all $\alpha \in A$, there exists $M(\alpha) \in \mathfrak{N}$ with $S \cap \alpha=M(\alpha) \cap \alpha$.

2.2. TheOREM. There exists a left-invariant extension $\left(G, \mathfrak{T}^{+}, \lambda^{+}\right)$of $\lambda$ such that every union of left cosets of $K$ is $\lambda^{+}$-measurable. If $K$ is a normal subgroup of $G$, the extension is invariant. 
Proof. Define $\mathfrak{R}^{+}$as in 2.1. It is clear that $\Re \subset \mathfrak{R}^{+}$, and it is easily verified that $\mathfrak{N}^{+}$is a $\sigma$-algebra which is closed under left translation and which contains every union of left cosets of $K$. (In fact, $\mathfrak{R}^{+}$is the smallest such algebra.)

Further, if $K$ is normal in $G$, it is easy to see that $\mathfrak{R}^{+}$is closed under righttranslation and inversion. Let $S$ be a member of $\mathfrak{R}^{+}$. If $S$ is not $\sigma$-finite, define $\lambda^{+} S=\infty$. If $S \in \mathbb{R}_{\sigma}^{+}$, we have $S=\cup M(\alpha) \cap \alpha$ for some subfamily $\{M(\alpha): \alpha \in A\}$ of $\mathbb{R}_{\sigma}$. Let us further require that $M(\alpha) \subset \operatorname{cl}(\alpha)$ for each $\alpha \in A$. By 1.8 and 1.11(3), each $M(\alpha)$ is uniquely defined up to measure zero, and $\lambda(M(\alpha))=0$ for all but countably many $\alpha$. Define

$$
\lambda^{+} S=\frac{1}{n} \sum_{\alpha \in A} \lambda(M(\alpha))
$$

(where $n$ is the index defined in 2.1).

Henceforth, given $S \in \Re^{+}$and $\alpha \in A, S(\alpha)$ is a $\lambda$-measurable subset of $\operatorname{cl}(\alpha)$ such that $S \cap \alpha=S(\alpha) \cap \alpha$ and $\lambda^{+}(S \cap \alpha)=n^{-1} \lambda S(\alpha)$; define $S(\alpha)$ to be $\varnothing$ if $S \cap \alpha=\varnothing$.

It remains to see that $\lambda^{+}$is a countably additive extension of $\lambda$; it suffices to show that $\lambda^{+} \mid \Re_{\sigma}^{+}$is countably additive and, since $\lambda$ is countably additive and both measure are left-invariant, that $\lambda^{+}(M \cap \operatorname{cl}(K))=\lambda(M \cap \operatorname{cl}(K))$ for $M \in \Re_{\sigma}$. To show countable additivity, let $S=\cup S_{i}$ where $\left\{S_{i}: i\right.$ $=1,2, \ldots\}$ is a pairwise disjoint sequence in $\Re_{\sigma}^{+}$. Since $S_{i}(\alpha)=\varnothing$ for all but countably many pairs $(i, \alpha)$ and, for a given $\alpha, \lambda\left(S_{i}(\alpha) \cap S_{j}(\alpha)\right)=0$ if $i \neq j$, we have

$$
\begin{aligned}
\lambda^{+} S & =\frac{1}{n} \sum_{\alpha} \lambda(S(\alpha))=\frac{1}{n} \sum_{a} \sum_{i} \lambda\left(S_{i}(\alpha)\right) \\
& =\frac{1}{n} \sum_{i} \sum_{\alpha} \lambda\left(S_{i}(\alpha)\right)=\sum_{i} \lambda^{+} S_{i} .
\end{aligned}
$$

Finally, if $M \in \Re_{\sigma}$, then

$$
\begin{aligned}
\lambda^{+}(M \cap \operatorname{cl}(K)) & =\sum_{j=1}^{n} \lambda^{+}\left(M \cap h_{j} K\right)=\sum \frac{1}{n} \lambda(M \cap \operatorname{cl}(K)) \\
& =\lambda(M \cap \operatorname{cl}(K)) .
\end{aligned}
$$

2.3. Corollary. Let $K_{1}$ be a subgroup of $G$, satisfying the conditions of Theorem 1.5. Then there exists an invariant extension $\left(G, 9 \pi^{+}, \lambda^{+}\right)$such that every union of translates of $K_{1}$ is $\lambda^{+}$-measurable.

Proof. Let $K$ be a relatively large normal subgroup of $G$ with $K \subset K_{1}$. Let $\left(G, \Re^{+}, \lambda^{+}\right)$be an invariant extension of $\lambda$, defined for $K$ as in 2.2. It is clear 
that every translate of $K_{1}$ is a union of cosets of $K$.

2.4 Notes. (1) We observe that Corollary 2.3 applies to all large subgroups of $G$, and to all relatively large subgroups of $G$ if $G$ happens to be compact or Abelian, or if every open subgroup of $G$ has finite index. It is tempting to speculate whether the conclusion of 2.3 holds if $J$ is any relatively large subgroup whose closure contains an open normal subgroup of $G$, or if $G$ is any unimodular group.

(2) In the class of all (hypothetical) left-invariant extensions of $\lambda,\left(G, \mathfrak{N}^{+}\right.$, $\lambda^{+}$) is uniquely determined as follows:

Lemma. Let $\left(G, \mathfrak{R}^{+}, \mu\right)$ be a left-invariant extension of $\lambda, \mathfrak{N}^{+}$being defined as in 2.1. Suppose, for all $M \in \mathfrak{K}_{\sigma}$ and all $x \in \operatorname{cl}(K)$,

$$
\mu(x M \cap K)=\mu(M \cap K) .
$$

Then $\mu=\lambda^{+}$.

Proof. Note that $\lambda^{+}$has the given property, for

$$
\lambda^{+}(x M \cap K)=n^{-1} \lambda\left((x M \cap \operatorname{cl}(K))=\lambda^{+}(M \cap K) .\right.
$$

Suppose that $\mu$ has the property. By 1.12, $\mu S=\infty=\lambda^{+} S$ for all $S$ $\in \Re^{+} \backslash \Re_{\sigma}^{+}$. If $S \in \Re_{\sigma}^{+}$, then $S(\alpha)=\varnothing$ for all but countably many $\alpha$; it suffices to show that $\mu[S(\alpha) \cap \alpha]=\lambda^{+}[S(\alpha) \cap \alpha]$ for each $\alpha$. Choose $g \in \alpha$; then

$$
\begin{aligned}
\lambda(S(\alpha)) & =\mu(S(\alpha))=\sum_{j=1}^{n} \mu\left(S(\alpha) \cap g h_{j} K\right)=\sum \mu\left(h_{j}^{-1} g^{-1} S(\alpha) \cap K\right) \\
& =\sum \mu\left(g^{-1} S(\alpha) \cap K\right)=n \mu(S(\alpha) \cap g K)=n \mu(S \cap \alpha) .
\end{aligned}
$$

Thus $\mu(S \cap \alpha)=n^{-1} \lambda(S(\alpha))=\lambda^{+}(S \cap \alpha)$.

2.5. Note. Given $M \in \mathfrak{N}$ with $0<\lambda M<\infty$,

$$
\lambda^{+}(x M \cap K)=n^{-1} \lambda(x M \cap \operatorname{cl}(K))
$$

for all $x \in G$. Thus the function $x \rightarrow \lambda^{+}(x M \cap K)$ is continuous; 2.4 implies that $\lambda^{+}$is the only countably additive left-invariant extension of $\lambda$, defined on $\mathfrak{R}^{+}$, for which this is true. This motivates the following

Definition. $\left(G, \Re^{+}, \lambda^{+}\right)$(as defined in 2.1-2.2) is called the continuous extension of $\lambda$ with respect to $K$.

\section{Families of relatively large subgroups.}

3.1 Notation and definitions. Given an indexed family $\left\{K_{\iota}: \iota \in I\right\}$ of relatively large subgroups of $G$, we write $\left(G, \mathscr{N}_{l}^{+}, \lambda_{l}^{+}\right)$for the continuous extension (as defined in 2.2 and 2.5) of $\lambda$ with respect to $K_{\iota}(\iota \in I)$. 
If $\mu$ is a finitely additive measure defined on an algebra $\Re$ of subsets of $G$, such that $\Re \subset \Re, \mu$ is an extension of $\lambda$, and $\mu(x S)=\mu(S)$ for all $S \in \Re$ and $x \in G$, then we say that $(G, \Re, \mu)$ is a finitely-additive left-invariant extension of $\lambda$.

3.2. Lemma. Let $K_{1}$ and $K_{2}$ be relatively large subgroups of $G$ with $K_{1} \subset K_{2}$. Then $\mathfrak{R}_{2}^{+} \subset \mathfrak{N}_{1}^{+}$and $\lambda_{1}^{+} S=\lambda_{2}^{+} S$ for all $S \in \mathfrak{R}_{2}^{+}$.

Proof. There is nothing to do unless $K_{1}$ is nonopen and $K_{1} \varsubsetneqq K_{2}$. Since $K_{2} \in \Re_{1}^{+}$, it is clear that $\Re_{2}^{+} \subset \Re_{1}^{+}$. To show that $\lambda_{1}^{+} \mid \Re_{2}^{+}=\lambda_{2}^{+}$, it suffices (by 2.4) to show that $\lambda_{1}^{+}\left(M \cap K_{2}\right)=\lambda_{1}^{+}\left(x M \cap K_{2}\right)$ for all $M \in \Re_{\sigma}$ $\cap \mathcal{P}\left(\operatorname{cl}\left(K_{2}\right)\right)$ and $x \in \operatorname{cl}\left(K_{2}\right)$.

Let $B=\left\{\alpha \in K_{2} / K_{1}:(M \cup x M) \cap \alpha \neq \varnothing\right\}$. ( $B$ is countable since $M$ $\cup x M$ is $\sigma$-finite.) Then

$$
\lambda_{1}^{+}\left(x M \cap K_{2}\right)=\sum_{\alpha \in B} \lambda_{1}^{+}(x M \cap \alpha)=\sum_{\alpha} \frac{1}{n_{1}} \lambda[x M \cap \operatorname{cl}(\alpha)]
$$

where $n_{1}=\left[\operatorname{cl}\left(K_{1}\right): K_{1}\right]$. Similarly,

$$
\lambda_{1}^{+}\left(M \cap K_{2}\right)=\sum_{\alpha} \frac{1}{n_{1}} \lambda[M \cap \mathrm{cl}(\alpha)] .
$$

Let $F$ be any finite subset of $B$, and $K_{F}=\cap_{\alpha \in F} \mathrm{cl}\left(\alpha \alpha^{-1}\right)$; since this is an open subgroup of $G$, we have $K_{2} \cap K_{F} x \neq \varnothing$. Let $x=h g$ where $h \in K_{F}$ and $g \in K_{2}$. Then

$$
\begin{aligned}
\sum_{\alpha \in F} \lambda[x M \cap \operatorname{cl}(\alpha)] & =\sum \lambda\left[g M \cap \operatorname{cl}\left(h^{-1} \alpha\right)\right]=\sum \lambda[g M \cap \operatorname{cl}(\alpha)] \\
& =\sum \lambda\left[M \cap \operatorname{cl}\left(g^{-1} \alpha\right)\right]=\sum n_{1} \lambda_{1}^{+}\left(M \cap g^{-1} \alpha\right) \\
& \leqslant n_{1} \lambda_{1}^{+}\left(M \cap K_{2}\right) .
\end{aligned}
$$

Thus $\lambda_{1}^{+}\left(x M \cap K_{2}\right) \leqslant \lambda_{1}^{+}\left(M \cap K_{2}\right)$; but by the same reasoning, since $M$ $\cap K_{2}=x^{-1} x M \cap K_{2}$, we have $\lambda_{1}^{+}\left(M \cap K_{2}\right) \leqslant \lambda_{1}^{+}\left(x M \cap K_{2}\right)$.

3.3. TheOREM. Let $\mathscr{K}=\left\{K_{\iota}: \iota \in I\right\}$ be any family of relatively large subgroups of $G$. Then there is a finitely additive left-invariant extension $\left(G, \Re_{I}^{+}, \lambda_{I}^{+}\right)$ of $\lambda$ such that

(1) $\mathscr{K} \subset \Re_{I}^{+}$;

(2) $\Re_{I}^{+}$is closed under right translation;

(3) given any finite subcollection $\mathscr{F}$ of $\mathscr{K}$, and $K=\cap \mathscr{F}, \lambda_{I}^{+}$is an extension of the continuous extension of $\lambda$ with respect to $K$;

(4) if each member of $\mathcal{K}$ contains a relatively large normal subgroup of $G, \mathfrak{R}_{I}^{+}$ is closed under inversion. 
PRoof. Let $\mathscr{K}_{0}=\left\{K_{\iota}: \iota \in I_{0}\right\}$ be a collection of relatively large subgroups of $G$ such that $\mathscr{K} \subset \mathscr{K}_{0}, \mathscr{K}_{0}$ is closed under conjugation and finite intersections, and every relatively large normal subgroup of $G$ is a member of $\mathscr{K}_{0}$. For each $\iota \in I_{0}$, let $\left(G, \Re_{\imath}^{+}, \lambda_{\imath}^{+}\right)$be the continuous extension of $\lambda$ with respect to $K_{\iota}$. Let $\Re_{I}^{+}=\bigcup_{\iota} \in I_{0} \Re_{\imath}^{+}$. Given any $\iota, \kappa \in I_{0}$, there exists $\nu \in I_{0}$ such that

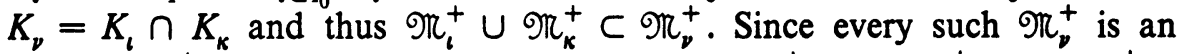
algebra, $\Re_{I}^{+}$is also an algebra. For every $S \in \Re_{I}^{+}$, define $\lambda_{I}^{+} S$ to be $\lambda_{l}^{+} S$, where $\iota$ is any element of $I_{0}$ such that $S \in \Re_{\imath}^{+}$. To show that $\lambda_{I}^{+}$is finitely additive, let $\left\{S_{j}: j=1,2, \ldots, m\right\}$ be a finite, pairwise disjoint subfamily of $\Re_{I}^{+}$. Let $S=\cup S_{j}$. Choose $\{\imath(j): j=1,2, \ldots, m\} \subset I_{0}$ so that $S_{j} \in \Re_{i(j)}^{+}$ for each $j$. Let $K_{\nu}=\bigcap_{j=1}^{m} K_{\iota(j)}$. By 1.4, $S \in \mathfrak{R}_{\nu}^{+}$, and by 3.2, $\lambda_{I}^{+} S_{j}=\lambda_{\nu}^{+} S_{j}$ for each $j$, and thus $\lambda_{I}^{+} S=\lambda_{\nu}^{+} S=\sum \lambda_{\nu}^{+} S_{j}=\sum \lambda_{I}^{+} S_{j}$. It is clear that $\lambda_{I}^{+}$is left-invariant and $\mathcal{K} \subset \Re_{I}^{+}$; it remains to show that $\left(G, \Re_{I}^{+}, \lambda_{I}^{+}\right)$satisfies conditions (2)-(4).

To prove that $\Re_{I}^{+}$is closed under right translation, suppose $S \in \Re_{I}^{+}$; then $x^{-1} S x \in \Re_{I}^{+}$also, so $S x \in \Re_{I}^{+}$.

Given $\mathscr{F}$ as in (3), let $K_{v}=\cap \mathscr{F}, \lambda^{+}=\lambda_{\nu}^{+}$, and $\mathfrak{R}^{+}=\Re_{v}^{+}$to obtain the desired extension (namely $\left(G, \Re^{+}, \lambda^{+}\right)$) of $\lambda$.

Finally, if $S \in \Re_{\imath}^{+}$where $K_{\imath}$ is a normal member of $\mathscr{K}_{\text {, then }} S^{-1} \in \Re_{\imath}^{+}$ also (by the second sentence in the statement of 2.2). This implies statement (4) of the present theorem.

3.4. The last result of this section is a counterexample, suggested by (3.6)-(3.7) of [3].

THEOREM. Let $G$ be an infinite compact Abelian torsion group. Let $\mathfrak{K}$ $=\left\{K_{\iota}: \iota \in I\right\}$ be the family of all large subgroups of $G$, and define $\left(G, \Re_{I}^{+}, \lambda_{I}^{+}\right)$ as in the previous theorem. Then $\lambda_{I}^{+}$is not countably additive and $\Re_{I}^{+}$is not a $\sigma$ algebra.

PRoof. Let $\Re$ be the $\sigma$-algebra generated by $\pi_{I}^{+}$. According to $(16.13 \mathrm{c})$ of [2], there is a subgroup $J$ of $G$ such that $G / J$ is countably infinite. Since $0<\lambda G<\infty$, it is impossible for $J$ to be measurable with respect to any countably additive translation-invariant extension of $\lambda$.

Let $\Phi$ be the group of all characters on $G$. We may take $\Phi$ to be the (compact) dual group of the discrete group $G_{d}$. By (25.9) of [2], $G$ is of bounded order, and $\Phi$ is therefore torsion, which is to say that the kernel of any element of $\Phi$ is a member of $K_{0}$. The dual group of $G_{d} / J$ is topologically isomorphic to a compact, metrizable (and therefore separable) subgroup of $\Phi$, namely $A(J)=\{\phi \in \Phi: \phi(x)=1$ for all $x \in J\}$. Let $\Delta$ be a countable dense subset of $A(J)$; then

$$
J=\{x \in G: \phi(x)=1 \text { for all } \phi \in \Delta\}=\bigcap_{\phi \in \Delta} K_{\phi},
$$


where $K_{\phi}=\phi^{-1}(1)$. (See (23.17), (24.15), (23.25), (23.24), and (24.10) of [2].) Thus $J \in \Re$; however, we have seen that it is impossible for $J$ to be $\lambda_{l}^{+}$measurable for any $\iota \in I$, thus $\Re \neq \cup \mathfrak{R}_{\imath}^{+}=\mathfrak{R}_{I}^{+}$, and $\mathfrak{R}_{I}^{+}$is not a $\sigma$ algebra.

Finally, suppose $\lambda_{I}^{+}$is countably additive. Then (by the Hopf Extension Theorem, (10.36) of [4]) there is a countably additive invariant extension $\mu$ of $\lambda_{I}^{+}$defined on $\Re$; but this is impossible since $J \in \Re$.

3.5. Question. Is $\lambda_{I}^{+}$countably additive if all the members of $\mathscr{K}$ are conjugate to each other?

\section{4. $\lambda^{+}$as an induced measure.}

4.1. Background. In this section, an alternate method is shown for obtaining the continuous extension of $\lambda$ with respect to a relatively large normal subgroup of $G$, as follows: $G$ is embedded (as a nonmeasurable subgroup having full outer measure in its closure) in another locally compact group $H$ in such a way that an invariant extension of $\lambda$ can be induced by extending Haar measure on $H$ to subsets of $G$. Specifically, in 4.2 (below) we find that the conditions stated in (2.2) of [3] can be imposed, or rather, approximated closely enough that the proof which appears loco citato remains valid.

In particular, the theorem in [3] is stated only for Abelian groups, although the authors observe (earlier in the same work) that it could easily be generalized. As an existence theorem, the subsequent application of (2.2) of [3] in its context depends on the commutativity of $G$ (as the dual of its character group), whereas in the present paper we have already established the existence of the desired measure by other means. The theorem may also be applied as a means of extending the methods of harmonic analysis to $\left(G, \Re^{+}, \lambda^{+}\right)$, via the relationship between $\lambda^{+}$-measurable functions on $G$ and Haar-measurable functions on $H$. This is discussed at length (for the Abelian case) in [3, §2], and, for the present special case, briefly noted in 4.4 below; 4.5 shows how our $\lambda^{+}$-measurable functions can be related to Haar-measurable functions on $G$ as well.

4.2. THEOREM (Part I). Let $K$ be a relatively large normal subgroup of $G$; let $H=G \times(G / K)=G \times A$ where the quotient group has the discrete topology. Define $\nu(g)=(g, g K)$ for all $g \in G$. Then

(1) $\nu$ is an open monomorphism of $G$ onto a relatively large subgroup of $H ; \nu(G)$ therefore has full outer measure in its closure.

(2) $\nu(G)$ is open [respectively, dense] in $H$ if and only if $K$ is open [dense] in $G$.

(3) If $S$ has compact $\left[\sigma\right.$-compact] closure in $G, \mathrm{cl}_{H}(\nu(S))$ is compact $[\sigma$ compact].

(4) If $U$ is an open subgroup of $G, \mathrm{cl}_{H}(\nu(U))$ is an open subgroup of $H$. 
(Part II). Let $\left(G, \mathfrak{I}^{+}, \lambda^{+}\right)$be defined as in $\$ 2$ (above). Define left Haar measure on $H$ to be $\lambda_{H}=1 / n(\lambda \times \mu)$ where $\mu$ is counting measure on $A$ (and, as before, $n=[\mathrm{cl}(K): K])$. Then

(5) $S \in \mathfrak{N}^{+}\left[S \in\left(\mathfrak{N}^{+}\right)_{\sigma}\right]$ if and only if $\nu(S)=M \cap \nu(G)$ for some $M$ $\in \mathfrak{R}_{H}\left[M \in\left(\Re_{H}\right)_{\sigma}\right]$.

(6) If $\nu(S)=M \cap \nu(G)$ and $M \in\left(\Re_{H}\right)_{\sigma}$, then $\lambda^{+} S=\lambda_{H} M$.

Proof. I. For any $S \subset G$,

(i) $\nu(S)=\cup_{\alpha \in A}(S \cap \alpha) \times\{\alpha\}$, and

(ii) $\operatorname{cl}_{H}(\nu(S))=\cup \operatorname{cl}(S \cap \alpha) \times\{\alpha\}$.

[As before, $A=G / K$.]

Let $L=\mathrm{cl}(K) \times\{K\}$. According to (ii), $L=\mathrm{cl}_{H}(\nu(K))$. Clearly $L$ is open, and thus $\operatorname{cl}_{H}(\nu(G))$ is also. Further, $\operatorname{cl}_{H}(\nu(G))=\nu(G) \cdot L$ and $\nu(G) \cap L$ $=K \times\{K\}$. Since $[\nu(G) \cdot L: \nu(G)]=[\operatorname{cl}(K): K]=n$, (1) follows.

(2) follows directly from (ii).

Since a compact [ $\sigma$-compact] subset of $G$ intersects only finitely many [countably many] members of $A$, (3) follows from (ii).

Finally, if $U$ is an open subgroup of $G$, each $\operatorname{set} \operatorname{cl}(U \cap \alpha)$ is open, since $U \cap \alpha$ is either void or a coset of the relatively large subgroup $U \cap K$, and thus (4) follows from (ii).

II. Suppose $M \subset H$; for each $\alpha \in A$, define $M_{\alpha}=\{x \in G:(x, \alpha) \in M\}$. Then $\nu(S)=M \cap \nu(G)$ if and only if $S \cap \alpha=M_{\alpha} \cap \alpha$ for all $\alpha \in A$. Since $A$ is discrete, $M \in \Re_{H}$ if and only if $M_{\alpha} \in \mathfrak{N}$ for all $\alpha$. This implies the relationship between $\mathfrak{R}_{H}$ and $\mathfrak{R}^{+}$stated in (5). Statement (4) (above) implies that $\nu(S)$ is $\sigma$-finite whenever $S$ is $\sigma$-finite. Suppose $\nu(S)=M \cap \nu(G)$; if we assume (as we may) that $M \subset \mathrm{cl}_{H}(M \cap \nu(G))$, then $M$ is $\sigma$-finite also. This completes the proof of (5). Statement (6) follows from 2.4(2).

4.3. Comment. If, in the statement of (2.2) of [3], we were to delete the word "Abelian" and substitute the phrase "having full outer measure in its closure" for "of full outer measure", we would have the statement of a theorem with almost exactly the same proof as the original; it is sufficient to observe that the term "invariant extension" has been suitably defined in $\$ 1$ of this paper and that $\lambda_{H}$ is an extension of Haar measure on $\mathrm{cl}_{H}(\nu(G))$. Part I of 4.2 shows that $G, H$, and $\nu$, as defined, satisfy the hypotheses of the (rewritten) theorem, while Part II shows that the measure space $\left(G, \mathfrak{T}^{+}, \lambda^{+}\right)$obtained is identical to the measure space $\left(G, \Re^{+}, \lambda^{+}\right)$, with $K$ normal in $G$, defined in $\S 2$ of this paper. Our next result follows immediately from part (iii) of (2.2) of [3] and (2.4) of [3]:

4.4. Corollary. Let $G, H, \nu$, and $\lambda^{+}$be defined as in 4.2 (and 2.2, above). Then

(1) $A$ character $\phi$ on $G$ is $\lambda^{+}$-measurable if and only if $\phi=\chi \circ \nu$ for some continuous character $\chi$ on $H$. 
(2) A nonnegative function $f$ on $G$ is $\lambda^{+}$-measurable if and only if there is a $\lambda_{H^{-}}$ measurable function $h$ such that

(a) $f=h \circ \nu$;

(b) $h$ vanishes off a $\sigma$-finite subset of $H$ whenever $f$ vanishes off a $\sigma$-finite subset of $G$;

(c) $\int f^{p} d \lambda^{+}=\int h^{p} d \lambda_{H}$ for all $0<p<\infty$.

4.5. Corollary. Let $G, K$, and $\lambda^{+}$be defined as in 2.2. Then

(1) A character $\phi$ on $G$ is $\lambda^{+}$-measurable if and only if $\phi=\gamma \psi$, where $\gamma$ and $\psi$ are characters on $G, \gamma$ is continuous, and $\psi(K)=\{1\}$.

(2) $A$ nonnegative function $f$ on $G$ is $\lambda^{+}$-measurable if and only if $f$ $=\sum_{\alpha \in A} h^{(\alpha)} I_{\alpha}$ where, for each $\alpha, I_{\alpha}$ is the characteristic function of $\alpha$ and $h^{(\alpha)}$ is a $\lambda$-measurable function. If $f$ vanishes off a $\sigma$-finite set, we may choose $\left\{h^{(\alpha)}: \alpha \in A\right\}$ so that each $h^{(\alpha)}$ vanishes off a $\sigma$-finite set, all but countably many $h^{(\alpha)}$ vanish identically, and

$$
\int f^{p} d \lambda^{+}=\frac{1}{n} \sum_{\alpha \in A} \int\left[h^{(\alpha)}\right]^{p} d \lambda \text { for all } 0<p<\infty .
$$

Proof. Each of statements (1) and (2) follows from the corresponding statement in 4.4. In (1), observe that continuous characters on $H$ are of the form $[\gamma, \psi]$, where $\gamma$ is continuous on $G, \psi$ annihilates $K$, and $[\gamma, \psi](x, y K)$ $=\gamma(x) \psi(y)$ for all $(x, y K) \in H$. Note that $[\gamma, \psi] \circ \nu=\gamma \psi$. In (2), let $h$ be a nonnegative $\lambda_{H}$-measurable function; for each $\alpha \in A$ and $x \in G$, define $h^{(\alpha)}(x)=h(x, \alpha)$ : it is obvious that $h \circ \nu=\sum h^{(\alpha)} I_{\alpha}$. By standard methods (see, e.g., $[2, \S 13]$ or $[4, \S 21])$, each of the functions $h^{(\alpha)}$ is $\lambda$-measurable, and, if $h$ vanishes off a $\sigma$-finite subset of $H$, then

$$
\int h^{p} d \lambda_{H}=\frac{1}{n} \iint[h(x, \alpha)]^{p} d \lambda(x) d \mu(\alpha)=\frac{1}{n} \sum_{\alpha \in A} \int\left[h^{(\alpha)}\right]^{p} d \lambda .
$$

(We observe that a subset $S$ of $H$ is $\sigma$-finite if and only if $\{\alpha \in A:(x, \alpha) \in S$ for some $x \in G\}$ is countable and, for each $\alpha,\{x \in G:(x, \alpha) \in S\}$ is $\sigma$ finite.)

\section{REFERENCES}

1. P. R. Halmos, Measure theory, Van Nostrand, Princeton, N.J., 1950. MR 11, 504.

2. E. Hewitt and K. A. Ross, Abstract harmonic analysis. Vol. I, Academic Press, New York; Springer-Verlag, Berlin, 1963. MR 28 \# 158.

3. _- Extensions of Haar measure and of harmonic analysis for locally compact Abelian groups, Math. Ann. 160 (1965), 171-194. MR 32 \#4208.

4. E. Hewitt and K. R. Stromberg, Real and abstract analysis, Springer-Verlag, Berlin and New York 1965; reprinted with corrections, 1969. MR 32 \# 5826; 43 \#428.

5. A. Hulanicki, On subsets of full outer measure in products of measure spaces, Bull. Acad. Polon. Sci. Sér. Sci. Math. Astronom. Phys. 7 (1959), 331-335. MR 21 \# 5710. 
6. G. L. Itzkowitz, Extensions of Haar measure for compact connected Abelian groups, Bull. Amer. Math. Soc. 71 (1965), 152-156. MR 30 \# 188.

7. - Extensions of Haar measure for compact connected Abelian groups, Nederl. Akad. Wetensch. Proc. Ser. A 68 = Indag. Math. 27 (1965), 190-207. MR 31 \#3540.

8. S. Kakutani and K. Kodaira, A non-separable translation invariant extension of the Lebesgue measure space, Ann. of Math. (2) 52 (1950), 574-579. MR 12, 246.

9. S. Kakutani and J. C. Oxtoby, Construction of a non-separable invariant extension of the Lebesgue measure space, Ann. of Math. (2) 52 (1950), 580-590. MR 12, 246.

10. J. L. Kelley, General topology, Van Nostrand, Princeton, N.J., 1955. MR 16, 1136.

11. H. L. Peterson, Discontinuous characters and subgroups of finite index, Pacific J. Math. 45 (1973), 683-691. MR 47 \#5174.

12. J. J. Rotman, The theory of groups: An introduction, Allyn and Bacon, Boston, 1965. MR 34 \#4338.

13. N. Th. Varopoulos, $A$ theorem on the Bohr compactification of a locally compact Abelian group, Proc. Cambridge Philos. Soc. 61 (1965), 65-68. MR 30 \#173.

Department of Mathematics, Indiana University Northwest, GaRy, Indiana 46408 